\title{
Sound absorption of a micro-perforated plate backed by a porous material under high sound excitation: measurement and prediction
}

\author{
Rostand Tayong*, Thomas Dupont, Philippe Leclaire \\ Acoustics \& Environmental Hydroacoustics lab., Université libre de Bruxelles, B-1050 Brussels, Belgium \\ LRMA - DRIVE, Université de Bourgogne, 49 rue Mademoiselle Bourgeois, 58027 Nevers cedex, France \\ LRMA - DRIVE, Université de Bourgogne, 49 rue Mademoiselle Bourgeois, 58027 Nevers cedex, France \\ *Corresponding author E-mail: rtayongb@ulb.ac.be
}

\begin{abstract}
The sound absorption coefficient of perforated facings backed by porous materials is studied under high sound intensities in the absence of mean flow. The theoretical considerations are based on the equivalent fluid following the Johnson-Champoux-Allard approach and the use of the transfer matrix method. To take into account the high sound levels effects, the air flow resistivity of each layer is modified following the Forchheimer law. Two specimens of perforated plate are built and tested when backed by a polymeric foam and a fibrous material. A specific impedance tube setup is developed for the measurement of the surface acoustic impedance for sound pressure levels ranging from $90 \mathrm{~dB}$ to $150 \mathrm{~dB}$ at the surface of the perforated facing. To corroborate the validity of the presented method, two considerations are particularly depicted in the experimental results: first, the case where the perforated facing and the porous material are both directly backed by a rigid wall and the case where there is an air cavity between the porous material and the rigid wall. Good agreement is observed between the simulation and the experimental results.
\end{abstract}

Keywords: Perforated facing, porous material, sound absorption coefficient, high sound level, Forchheimer.

\section{Introduction}

In practical noise control applications [1] related to architectural acoustic design, aircraft and automotive domain, perforated plates are very often used to protect porous materials. Under low sound pressure levels, such assemblies are known [2-4] to result in shifting the resonance frequency, which is mainly induced by the perforated plate, to lowfrequency bands and enhance the acoustic absorption near the resonance frequency. The absorption performance of perforated plates alone is known [5] to be enhanced when the perforation sizes approach the boundary layer thickness sizes (submillimetric sizes very often). Yet, in many of these applications, the incident sound pressure levels tend to be relatively high such that classical models [6,7] would only result in poor prediction. Therefore, how these assemblies behave under the nonlinear regime (high sound pressure levels) is valuable to be studied.

The acoustical properties of air-backed Micro-Perforated Plates (MPP) have been widely investigated analytically [5,811], numerically [12-14] and experimentally [9,13]. In the linear regime (low sound pressure levels) of such materials, viscous and thermal effects are known to be dominant $[9,15,16]$ and the incident pressure is proportional to the particle velocity through the perforations. Recently, Atalla and Sgard [11] used a rigid frame approach following the JohnsonChampoux-Allard [10] approach to predict the acoustic impedance of such materials. Under high sound pressure levels, from Ingard and Ising [15] and Melling [17] works, it is known that the acoustic resistance increases with the acoustic velocity in the perforations meanwhile the reactance relatively decreases. These observations are confirmed by other experimental works $[18,19]$.

The linear regime studies on acoustical properties of classical porous materials [10,20,21] have been extensively carried out and their behavior seems to be well mastered. Yet, there is very few literature dealing with the assemblies of perforated plates and porous materials under high sound excitations. This paper is devoted to experimentally determine, under high sound pressure levels, the acoustic absorption of MPP when backed by a porous material (polymeric foam and fiber material). For the theory, the rigid frame approach is described and applied for each sample and both cases of these assemblies directly in contact with a rigid wall and when there is an air gap before the rigid wall is modeled using the Transfer Matrix Method [10,22] (TMM). The nonlinear regime effects are taken into account by the use of Forchheimer law [23-25]. Since the MPP is known to be efficient in low and medium frequency ranges, the study is 
focused on the frequencies of $348 \mathrm{~Hz}$ and $442 \mathrm{~Hz}$. The predictions and the experimental results are compared and discussed in details. Figure 1 depicts some of the configurations considered in this study.

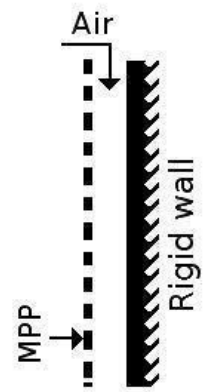

(a)

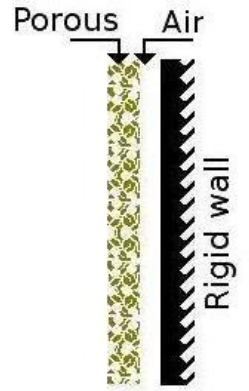

(b)

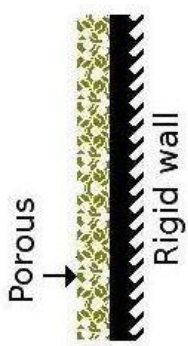

(c)

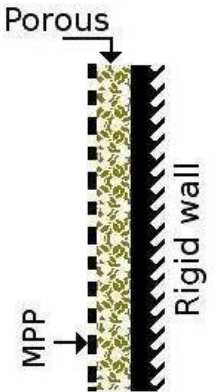

(d)

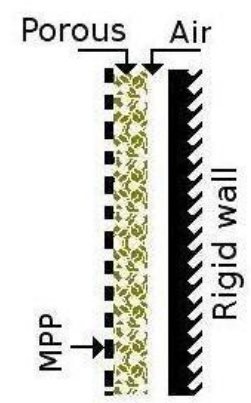

(e)

Fig. 1: Representative scheme of the different configurations tested. a) MPP backed by an air cavity before the rigid wall; b) Porous material with an air cavity before the rigid wall; c) Porous material directly backed by a rigid wall; d) MPP backed by a porous material with no air cavity before the rigid wall; e) MPP backed by a porous material with an air cavity before the rigid wall.

\section{Johnson-Champoux-Allard equivalent fluid model}

\subsection{Case of a perforated plate}

The acoustic impedance of a perforated plate is defined as the ratio of the parietal pressure difference across the plate (or the perforations) to the air particle velocity through the perforations. The specific acoustic impedance $Z_{1}$ of a perforated plate is then defined as

$$
Z_{1}=\frac{\Delta p}{u_{s}}=\frac{\Delta p}{\phi_{1} u_{1}}
$$

where $\Delta p$ is the pressure difference across the plate, $u_{s}$ is the superficial seepage velocity, $u_{1}$ is the particle velocity inside the perforations and $\phi_{1}$ is the perforated plate porosity (also known as the open area ratio for perforated plates). It is shown from Atalla and Sgard [11] work that a perforated plate can be modeled as an equivalent fluid following Johnson-Champoux-Allard [10] approach with an equivalent tortuosity which depends on the media surrounding the perforated plate. For acoustic wavelengths much larger than the plate thickness, the specific impedance of a perforated plate can be obtained by

$$
Z_{1}=\frac{1}{\phi_{1}} j \omega \tilde{\rho}_{e 1} h_{1}
$$

where $h_{1}$ is the perforated plate thickness, $\omega$ is the angular frequency and $\tilde{\rho}_{e 1}$ is the effective density of the perforated plate expressed as

$$
\tilde{\rho}_{e 1}=\rho_{0} \tilde{\alpha}_{1}
$$

where $\rho_{0}$ is the air density and $\tilde{\alpha}_{1}$ the dynamic tortuosity given by

with

$$
\tilde{\alpha}_{1}=\alpha_{\infty 1}\left(1+\frac{\sigma_{1} \phi_{1}}{j \omega \rho_{0} \alpha_{\infty 1}} G_{J 1}(\omega)\right)
$$

$$
G_{J 1}(\omega)=\left(1+j \frac{4 \omega \rho_{0} \alpha_{\infty 1}^{2} \eta}{\sigma_{1}^{2} \phi_{1}^{2} \Lambda_{1}^{2}}\right)^{\frac{1}{2}}
$$

and where $\sigma_{1}$ is the perforated plate resistivity $\left(\sigma_{1}=8 \eta / \phi_{1} \mathrm{r}^{2}\right)$ obtained from the static viscous permeability expression for the Poiseuille law (see Ref.[10]), $\rho_{0}$ the density of the fluid at rest, $\alpha_{\infty 1}$ is the geometrical tortuosity, $\eta$ is the dynamic viscosity of air, $\Lambda_{1}$ is the viscous characteristic length which is equal to the hydraulic radius of the pores. Due to the small thickness and shape of the pores, thermal effects are considered negligible. The coupling of the perforated plate with its surroundings modifies the length of the system. To account for this modification, the geometric tortuosity should be properly corrected. When backed by an air cavity, the tortuosity should be corrected (see Ref [11]) as

$$
\alpha_{\infty 1}=1+\frac{2 \varepsilon_{e}}{h_{1}}
$$

and

$$
\alpha_{\infty 1}=1+\frac{2 \varepsilon_{e}}{h_{1}}\left(1+\operatorname{Real}\left(\tilde{\alpha}_{2}\right)\right)
$$

when backed by a rigid frame porous layer, where $\operatorname{Real}\left(\tilde{\alpha}_{2}\right)$ is the real part of the dynamic tortuosity of the fibrous material, $\varepsilon_{e}=0.48 \sqrt{\pi r^{2}}\left(1-1.14 \sqrt{\phi_{1}}\right)$ and $\sqrt{\phi_{1}}<0.4$. 


\subsection{Case of a porous material}

The general expressions for the effective fluid density $\tilde{\rho}_{e 2}$ and the effective compressibility modulus of the fluid are given in Ref.[10] and written as

$$
\tilde{\rho}_{e 2}(\omega)=\alpha_{\infty 2} \rho_{0}\left[1+\frac{\sigma_{2} \phi_{2}}{j \omega \rho_{0} \alpha_{\infty 2}} G_{J 2}(\omega)\right]
$$

and

$$
K_{2}(\omega)=\gamma P_{0} /\left[\gamma-(\gamma-1)\left(1+\frac{\sigma_{2} \phi_{2}}{j B^{2} \omega \rho_{0} \alpha_{\infty 2}} G_{J 2}^{\prime}\left(B^{2} \omega\right)\right)^{-1}\right]
$$

where

$$
G_{J 2}(\omega)=\left(1+j \frac{4 \omega \rho_{0} \alpha_{\infty 2}^{2} \eta}{\sigma_{2}^{2} \phi_{2}^{2} \Lambda_{2}^{2}}\right)^{1 / 2}
$$

with

$$
G_{J 2}^{\prime}\left(B^{2} \omega\right)=\left(1+j \frac{4 \omega B^{2} \rho_{0} \alpha_{\infty 2}^{2} \eta}{\sigma_{2}^{2} \phi_{2}^{2} \Lambda_{2}^{2}}\right)^{1 / 2}
$$

and

$$
\sigma_{2}^{\prime}=\frac{8 \alpha_{\infty 20} \eta}{\phi_{2} \Lambda_{2}^{\prime}}
$$

In the above expressions, $\gamma$ represents the adiabaticity constant (ratio of the specific heat at constant pressure onto the same quantity at fixed volume), $P_{0}$ the ambient pressure, $\mathrm{B}^{2}$ the Prantl number, $\phi_{2}$ the porosity of the porous material, $\alpha_{\infty 2}$ the tortuosity, $\sigma_{2}$ the air flow resistivity, $\eta$ the air viscosity, $\Lambda_{2}$ and $\Lambda_{2}^{\prime}$ the viscous and thermal characteristic lengths (also called shape factors).

The complex sound wavespeed $c_{2}$ inside the porous medium is then provided by

$$
c_{2}(\omega)=\sqrt{\frac{K_{2}(\omega)}{\tilde{\rho}_{e 2}(\omega)}},
$$

and the characteristic acoustical impedance $Z_{2}$ of the porous medium is then given by

$$
Z_{2}(\omega)=\sqrt{K_{2}(\omega) \tilde{\rho}_{e 2}(\omega)} .
$$

\subsection{The Transfer Matrix Method}

The Transfer Matrix Method (TMM) is a classical method used to determine the pressure fields reflected by or transmitted through a multi-layered material [22]. In this study, the TMM is used to calculate the surface impedance of a perforated plate backed by a porous material as depicted in figure 1 where, otherwise mentioned in this theoretical section, the subscript 1 (resp. 2) is used for the perforated plate (resp. the porous material). In its principle, the TMM consists in expressing the conserved physical data at one end of the layer as a function of the physical data at the other end.

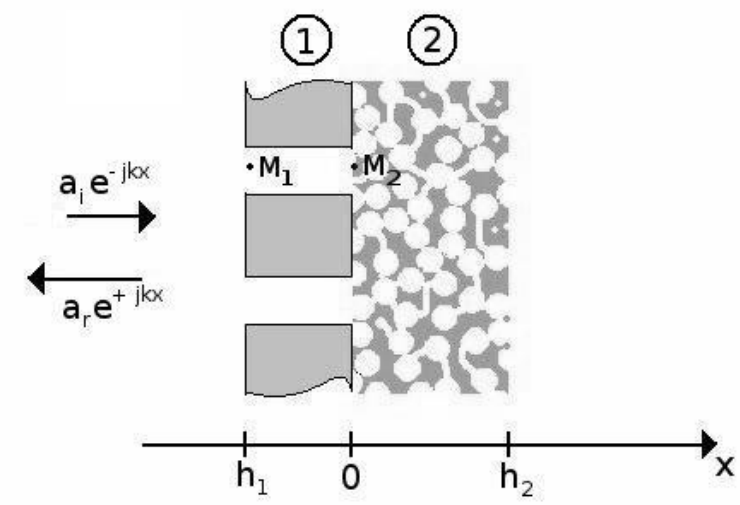

Fig. 1: Configuration of the two-layer material. Perforated plate (Region 1) backed by a rigid porous material (Region 2).

In the first step, the propagation in layer 1 (the perforated plate) is considered. Using the notations in Fig.1, expression (1) can be written as

$$
Z_{1}=\frac{p\left(-h_{1}^{+}\right)-p\left(0^{-}\right)}{\phi_{1} u\left(-h_{1}^{+}\right)}
$$

The continuity conditions must be applied at the interfaces $x=-h_{1}$ and $x=0$ to get the pressure and the velocity at the external sides. Considering the point $M_{1}$ at the interface at $x=-h_{1}$ but inside the layer 1 and the point $M_{2}$ at the interface at $x=0$ but inside the layer 2 , these conditions are given as 


$$
\begin{aligned}
& p\left(-h_{1}^{-}\right)=p\left(-h_{1}^{+}\right), \\
& p\left(0^{-}\right)=p\left(0^{+}\right), \\
& u\left(-h_{1}^{-}\right)=\phi_{1} u\left(-h_{1}^{+}\right), \\
& \phi_{1} u\left(0^{-}\right)=\phi_{2} u\left(0^{+}\right) .
\end{aligned}
$$

If one assumes that the velocity does not vary noticeably from one face to the other in the perforations, because of the small plate thickness, it can be shown that the transfer matrix $T_{1}$ describing the propagation in the perforated plate is written as

$$
T_{1}=\left(\begin{array}{cc}
1 & Z_{1} \\
0 & 1
\end{array}\right) .
$$

Following the same principle applied to layer 2 (that is the porous material), it can also be shown that the transfer matrix $\mathrm{T}_{2}$ is written as

$$
T_{2}=\left(\begin{array}{cc}
\cos \left(k_{2} h_{2}\right) & -j Z_{2} \sin \left(k_{2} h_{2}\right) \\
j \frac{1}{z_{2}} \sin \left(k_{2} h_{2}\right) & \cos \left(k_{2} h_{2}\right)
\end{array}\right) .
$$

In the case where a layer of airspace (layer 3 ) is considered behind the porous material and before the rigid wall, it can be shown that the transfer matrix of this layer of airspace is given by

$$
T_{3}=\left(\begin{array}{cc}
\cos \left(k_{0} h_{\text {cav }}\right) & j Z_{0} \sin \left(k_{0} h_{\text {cav }}\right) \\
j \frac{1}{Z_{0}} \sin \left(k_{0} h_{\text {cav }}\right) & \cos \left(k_{0} h_{\text {cav }}\right)
\end{array}\right)
$$

where $k_{0}$ is the wavenumber in the air and $h_{\text {cav }}$ is air cavity depth.

Once the matrices of each layer is obtained, the direct product is possible in order to get the global transfer matrix $\mathrm{T}$ of the assembly. The global transfer matrix $\mathrm{T}$ is obtained by

$$
[T]=\left[T_{1}\right] .\left[T_{2}\right]
$$

when the MPP and the porous material are directly backed by a rigid wall and by

$$
[T]=\left[T_{1}\right] \cdot\left[T_{2}\right] \cdot\left[T_{3}\right]
$$

when the MPP and the porous material are backed by an air cavity before the rigid wall. The surface impedance $Z_{s}$ of the global sample is then obtained by

$$
Z_{S}=\frac{T(1,1)}{T(2,1)} .
$$

The reflection coefficient $\mathrm{R}$ is obtained by

$$
R=\frac{z_{s}-z_{0}}{z_{s}+z_{0}}
$$

and the sound absorption coefficient $\alpha$ is given as

$$
\alpha=1-|R|^{2}
$$

\section{The high sound pressure regime effects}

On the macroscopic scale, fluid flow in a porous medium at low velocity is generally described by Darcy's law, which presents a linear relationship between the pressure gradient and the fluid velocity in the porous media. However, as the velocity is raised beyond a certain value, numerous observations [23,24] have confirmed that Darcy's law must be replaced by another empirical formula, the Forchheimer equation, to account for nonlinear effects. The Forchheimer parabolic law is written as

$$
\Delta p=F_{1} u_{s}^{2}+F_{2} u_{s},
$$

where $F_{1}$ and $F_{2}$ are constants evaluated from experimental data. Recent works $[25,29,30]$ on nonlinearity (high sound intensities regime) in rigid porous media lead to the conclusion that only resistivity is involved in the nonlinear effects. From Auregan and Pachebat [25] work, since it is proved that the resistivity increases linearly with the seepage velocity, the above Forchheimer law is also equivalent to 


$$
\sigma_{\text {nonlinear }}=\sigma C_{1} R e_{p}+\sigma\left(1+C_{2}\right) \text {, }
$$

where $\sigma_{\text {nonlinear }}$ is the nonlinear (high sound intensities regime) air flow resistivity, $\sigma$ is the linear (low sound intensities regime) air flow resistivity described in the previous section, $C_{1}$ and $C_{2}$ are the nonlinear coefficients and $R e_{p}$ is the Reynolds number inside the pores (case of porous material) or the perforations (case of MPP).

\section{Experimental setup}

The measurements are performed on steel perforated plates (MPP\#1 and MPP\#2), a polymeric foam (porous\#1) and a fibrous material (porous\#2). Each sample tested has an external diameter of $100 \mathrm{~mm}$ which corresponds to the inner diameter of the impedance tube. During the measurements of the assemblies, the perforated plates are directly in contact with the porous material. The samples characteristics are given in table 1 .

A schematic of the impedance tube used for the experiments is shown in Fig.2. It is a rigid circular plane-wave tube with a diameter of $100 \mathrm{~mm}$ (cut-off frequency of the tube is $1.7 \mathrm{KHz}$ ). On the left hand side, a JBL model 2450J compression driver is mounted as the excitation source. This compression driver is powered by a power amplifier. A transition piece provides the continuity transition between the circular section of the compression driver and the circular cross section of the impedance tube. On the right hand side of the tube, a soundproof plunger is used as the rigid backing wall.

Table 1: Samples characteristics and acoustical parameters (porosity $\phi$, resistivity $\sigma$, tortuosity $\alpha_{\infty}$, viscous characteristic length $\Lambda$, thermal characteristic length $\Lambda^{\prime}$ ). Porous\#1 is a polymeric foam and Porous\#2 is a fibrous material.

\begin{tabular}{|c|cc|cc|}
\hline & \multicolumn{2}{|c|}{ System\#1 } & \multicolumn{2}{c|}{ System\#2 } \\
\cline { 2 - 5 } & MPP\#1 & Porous\#1 & MPP\#2 & Porous\#2 \\
\hline Thickness h [mm] & 2.00 & 68.00 & 1.00 & 40.00 \\
Hole diameter d [mm] & 0.70 & - & 0.50 & - \\
$\phi[\%]$ & 1.94 & 99.00 & 0.52 & 95.00 \\
$\sigma\left[\right.$ N.s.m $\left.{ }^{-4}\right]$ & 61940 & 12000 & 452923 & 11700 \\
$\alpha_{\infty}$ & 1.25 & 1.001 & 1.39 & 1.260 \\
$\Lambda[\mu \mathrm{m}]$ & 350.00 & 230.00 & 250.00 & 80.00 \\
$\Lambda^{\prime}[\mu \mathrm{m}]$ & 350.00 & 250.00 & 250.00 & 240.00 \\
\hline
\end{tabular}

The sealing for the plunger is ensured using a rubber seal. By moving the plunger along the longitudinal axis of the tube, it is possible to create an air cavity behind the samples. Three $1 / 4^{\prime \prime}$ microphones are used to perform the signal detection. The first two microphones (micro 1 and 2 in Fig.2) are used to calculate the surface impedance of the samples by the two-microphone standing waves method (see Ref.[31] for more details). The distance between these microphones is $\mathrm{s}=50 \mathrm{~mm}$. And the distance between microphone 2 and the sample is $l_{2}=11 \mathrm{~mm}$. The third microphone (reference micro in Fig.2) is used to monitor the pressure level $\mathrm{L}_{\mathrm{p}}$ at the sample surface. When performing high sound pressure measurements inside an impedance tube, it is important to check the resulting standing wave. In fact, up to a certain level, depending on the frequency excitation and the sample parameters, the standing wave seems to saturate and therefore the linear propagation hypothesis would no longer be valid. Effects of bifurcation may also occur. These phenomena of saturation and bifurcation were observed and shown by Maa and Liu [32]. No saturation and no considerable bifurcation were observed at the resonance frequencies studied.

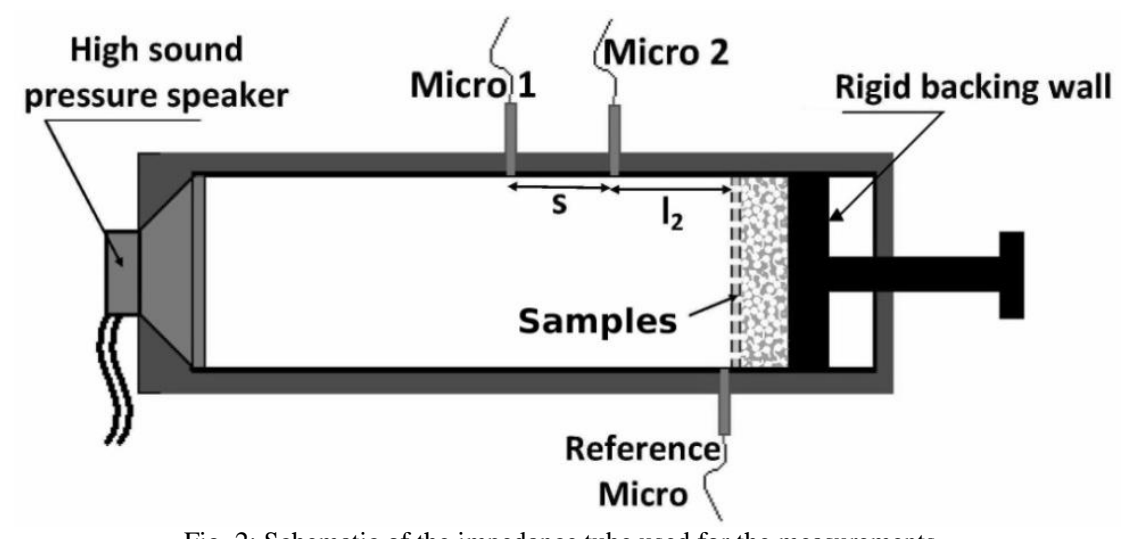

Fig. 2: Schematic of the impedance tube used for the measurements. 
The study is focused on the acoustical behavior of the assemblies for given frequencies. Since each system, formed by a perforated plate and a porous material with and without an air cavity behind, has different reactance, to compare the results at a given frequency, it is made use of different air cavity depth as followed:

- In a first step, the measurement are done for the case of the MPP coupled to the porous material and the resulted system directly backed by the rigid wall without any air cavity (fig.1d). This allows to obtain the resonance frequency $f_{0}$ of the system.

- In the next step, for the case of the MPP alone backed by an air cavity and the rigid wall (fig.1a), that is without the porous material, the air cavity depth is tuned so as to obtain the maximum of absorption at the frequency $f_{0}$.

- Finally, for the case of the MPP backed by the porous material with an air cavity before the rigid wall (fig.1e), half the porous material thickness is used and the air cavity depth before the rigid wall is tuned so as to obtain the first maximum of absorption at the frequency $f_{0}$.

The various air cavity depths and the resonance frequencies used in this study are given in table 2.

\begin{tabular}{|c|cc|cc|} 
Table 2: Air cavity depths and resonance frequencies of study. \\
\begin{tabular}{|c|ccccc|}
\hline & \multicolumn{2}{c|}{ Figure $1(\mathrm{a})$} & \multicolumn{2}{c|}{ Figure 1(e) } \\
\cline { 2 - 5 } & \multicolumn{1}{|c}{ MPP\#1 } & MPP\#2 & System\#1 & System\#2 \\
\hline Air cavity depth $[\mathrm{mm}]$ & 84 & 72 & 25 & 21 \\
Resonance frequency $[\mathrm{Hz}]$ & 442 & 348 & 442 & 348 \\
\hline
\end{tabular}
\end{tabular}

Assuming the plane wave hypothesis, one can measure the pressures and velocities on any section of the tube using the two-microphone method. See for instance Dalmont [33] for more details. The acoustic velocity on the panel surface is given by:

$$
u=j \frac{p_{1}}{Z_{0}} \frac{H \cos \left(k_{0} l_{1}\right)-\cos \left(k_{0} l_{2}\right)}{\sin \left(k_{0} s\right)}
$$

where $l_{1}=s+l_{2}$ as in Fig.2, $H$ is transfer function, $p_{1}$ is the pressure on microphone 1 , and $l_{1}$ (resp. $l_{2}$ ) is the distance from microphone 1 (resp. 2) to the panel sample.

Some deviations to Darcy's law are known to appear when the seepage velocity increases [27]. The relevant parameter to describe the impedance or the resistivity variations is the Reynolds number [25] defined as

$$
R e=\frac{2 \Lambda U}{v}
$$

with $U$ the mean velocity in front of the sample and $v$ the kinematic viscosity.

When a material of fixed thickness $\mathrm{h}$ is submitted to a difference of static pressure $\Delta P$, a macroscopic flow of average velocity $U$ appears through the material and the resistivity is defined by the ratio of static pressure difference to the product of the velocity and the thickness of the porous sample written as

$$
\sigma=\frac{\Delta P}{U h}
$$

The principle of the resistivity determination is based on low frequency asymptotic approximation of the LafargeAllard's model [10]. In the experimental results, the resistivity $\sigma_{1}$ of the perforated plate is given by

$$
\sigma_{1}=\frac{\operatorname{Real}\left\{\mathrm{Z}_{1}\right\}}{h_{1}}
$$

where Real $\left\{Z_{1}\right\}$ is the real part of the surface impedance of the perforated plate backed by an air cavity and $h_{1}$ is the thickness of the perforated plate.

\section{Results and discussion}

\subsection{Resistivity as a function of the inlet Reynolds number}

Figure 3 presents the experimental and linear fit results of the air flow resistivity of the samples as a function of the inlet Reynolds number. Figure $3 a$ ) is for the porous materials results and figure $3 b$ ) is for the MPP results obtained at the frequencies of $442 \mathrm{~Hz}$ (MPP\#1) and $348 \mathrm{~Hz}$ (MPP\#2). A linear fit applied to the high sound levels results (above 135 $\mathrm{dB}$ ) allows to obtain the Forchheimer coefficients of each sample. According to Forchheimer law [23,25,29], under high 
sound pressure regime, the resistivity of a porous material is linearly dependent with the inlet particle velocity (implicitly the Reynolds number). The obtained coefficients are listed in table 3.

\begin{tabular}{|l|cc|cc|}
\multicolumn{4}{|c}{ Table 3: Forchheimer coefficients for the samples. } \\
\cline { 2 - 5 } & \multicolumn{2}{|c|}{ System\#1 } & \multicolumn{2}{c|}{ System\#2 } \\
\cline { 2 - 5 } & MPP\#1 (442 Hz) & Porous\#1 & MPP\#2 (348 Hz) & Porous\#2 \\
\hline C1 & 0.3818 & 0.0127 & 1.7515 & 0.7541 \\
C2 & 1.6996 & 0.3926 & 1.2239 & 0.0550 \\
\hline
\end{tabular}
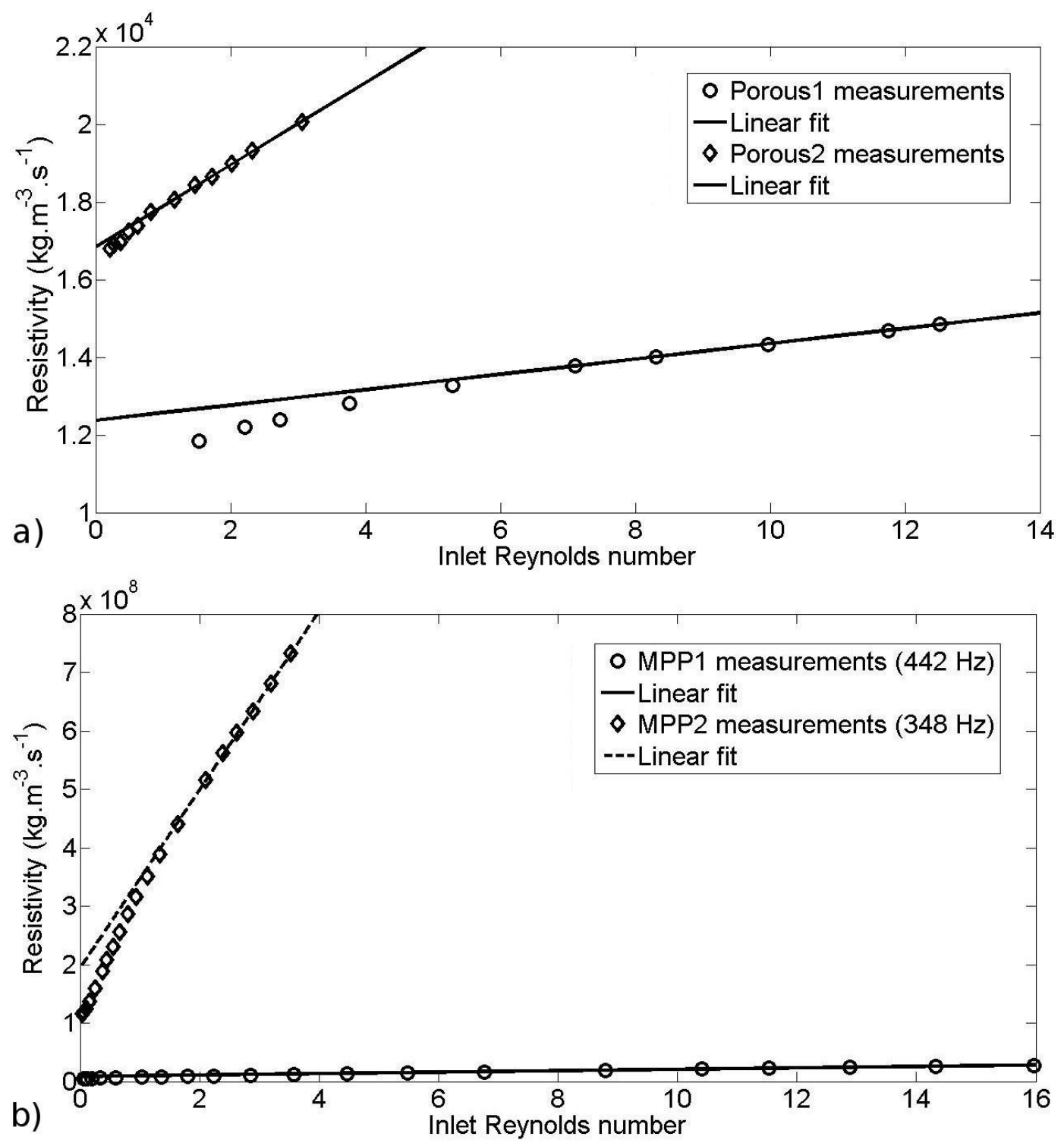

Fig. 3: Experimental and linear fit of the samples resistivities: a) Resistivity of the porous material (Porous1 and porous2) as a function of the inlet Reynolds number; b) Resistivity of the perforated plate (MPP\#1 and MPP\#2) as a function of the inlet Reynolds number.

\subsection{Surface resistance as a function of the inlet Reynolds number}

Figure 4 presents the results of the normalized surface resistance as a function of the inlet Reynolds number. In figure 4a) the experimental and calculated normalized surface resistance for the MPP backed by an air cavity are plotted as a function of the Reynolds number. In figure 4b) the experimental and calculated normalized surface resistance for the MPP backed by a porous material and a rigid wall (no air cavity) are plotted as a function of the Reynolds number. As expected, the normalized surface resistance is linearly dependent with the inlet Reynolds number (consequence of the 
Forchheimer law). Although the porous material increases the resistance result of the system, one can observe from both figures that the presence of a porous material behind the perforated plate slightly affect the slope of the curve.

According to Melling [17], in general, the high sound regime for the MPP (minute perforations) takes place around level of $120 \mathrm{~dB}$ in front of the perforations. This latter value is only indicative and strongly depends on the perforations size. For the presented results of the MPP, the levels of pressure upper than $135 \mathrm{~dB}$ are mainly considered. Measurements and prediction agree fairly well.
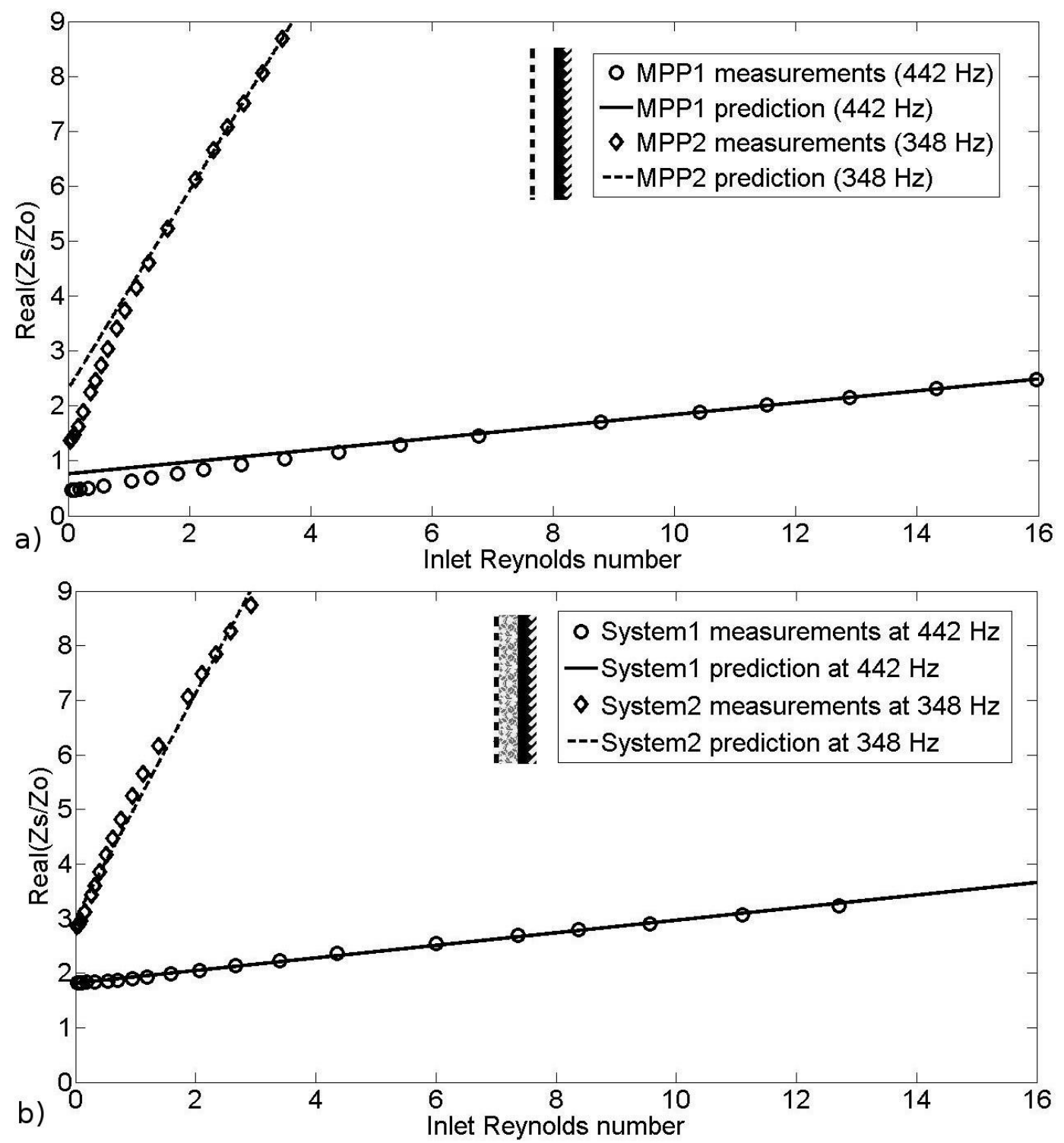

Fig. 4: Experimental and simulation results of the normalized surface resistance as a function of the inlet Reynolds number: a) MPP backed by an air cavity depth of $84 \mathrm{~mm}$ (MPP1) and $72 \mathrm{~mm}$ (MPP2). b) MPP backed by a porous material and a rigid wall (no air cavity).

\subsection{Maximum of absorption coefficient as a function of inlet Reynolds number}

Figure 5 presents the experimental and simulation results of the maximum of absorption coefficient as a function of inlet Reynolds number. In figure 5a) measurements and prediction for the MPP backed by an air cavity are plotted as a function of the Reynolds number. In figure 5b) measurements and prediction for the MPP backed by a porous material and a rigid wall (no air cavity) are plotted as a function of the Reynolds number. According to Tayong et al. [19], the maximum of absorption can be a positive or a negative function of the inlet flow velocity. Their work suggests the existence of a maximum of absorption as a function of flow velocity (explicitly the Reynolds number). This behavior is clearly observable in figure 5a) for MPP1 results. For this latter case, one can observe that the maximum of absorption increases with the inlet Reynolds number, reaches a maximum of 1 as the Reynolds number approaches its critical value $(\operatorname{Re} \approx 3.45)$ and then decreases for the Reynolds number increasing beyond this critical value. As shown by Tayong et al. [19], this behavior (also observed by Umnova et al. [29] for rigid-porous layers) can be observed if the sample 
resistance is less than the medium resistance and practically very often if the critical value is above the linear/nonlinear regime limit. In the case of MPP2, the maximum of absorption solely decreases with the increase of the Reynolds number inside the perforations (the linear/nonlinear regime limit is found to be in the linear regime). The simulation fairly predicts the critical Reynolds number. Both measurements and prediction are in good agreement for the high sound levels considered.

Figure 5b) presents the measurement and prediction results of the maximum of absorption for the case of the MPP backed by a porous material and a rigid wall (no air cavity) as a function of the inlet Reynolds number. In this figure, the maximum of absorption (cases of System\#1 and System\#2) solely decreases with the increase of the inlet Reynolds number. A comparison of these results with those of the MPP coupled to an air cavity and a rigid wall leads to the conclusion that the presence of the porous material behind the MPP causes a shift of the limit Reynolds number towards the low ranges regime. This is explained by the fact that the porous material behind the MPP increases the system resistance $[2,4]$ as stipulated earlier. The measurements and prediction are also in good agreement.
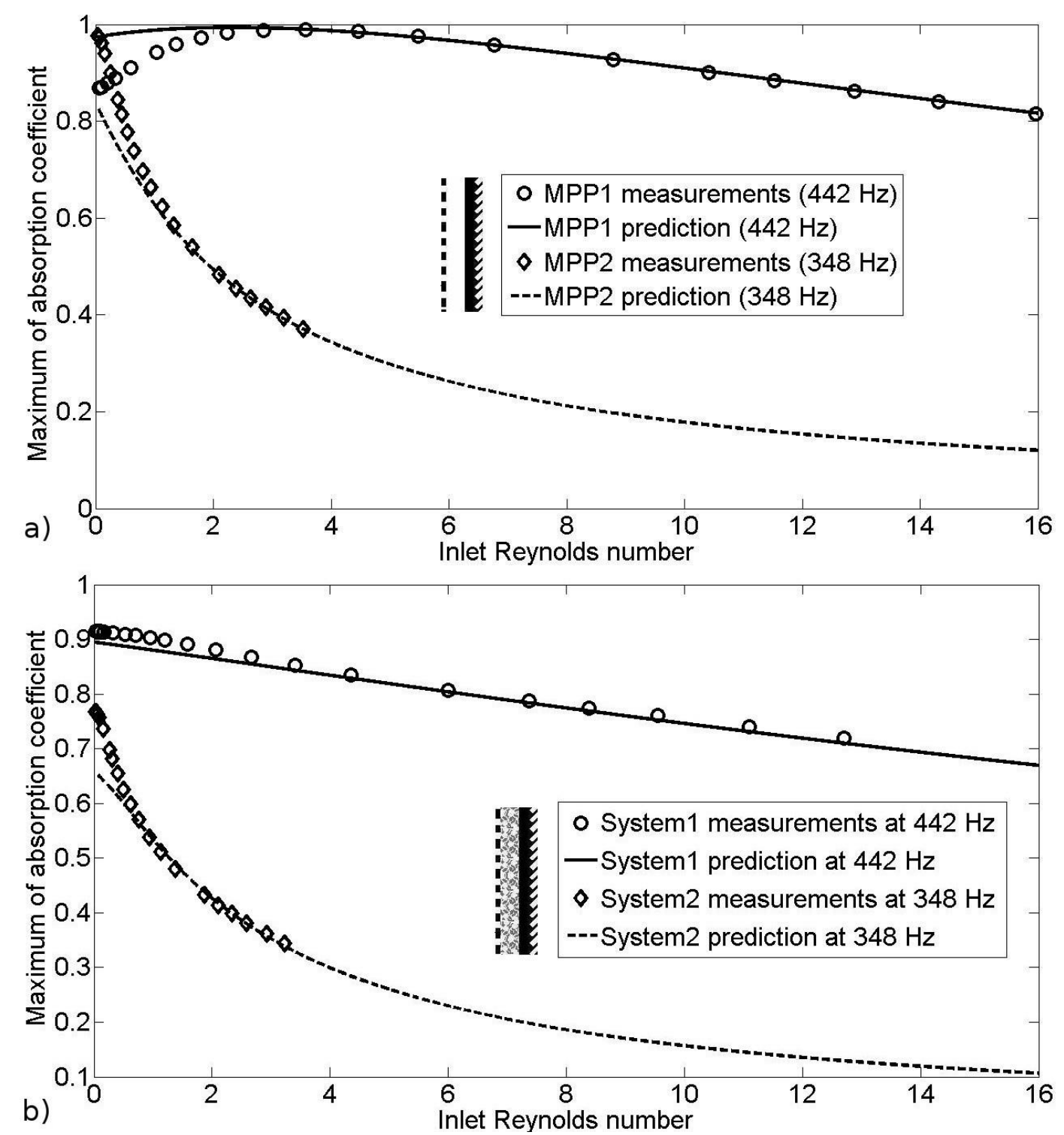

Fig. 5: Experimental and simulation results of the maximum of absorption as a function of the inlet Reynolds number: a) MPP backed by an air cavity depth of $84 \mathrm{~mm}$ (MPP1) and $72 \mathrm{~mm}$ (MPP2). b) MPP backed by a porous material and a rigid wall (no air cavity).

\subsection{Absorption coefficient as a function of frequency}

Figure 6 presents the results of the absorption coefficient results as a function of frequency for system\#1 with no air gap before the rigid wall for an incident pressure level $L_{p}$ of $145 \mathrm{~dB}$ (corresponding to $R e=8.381$ ). One can observe that the simulation properly predicts the measurements. Far beyond the resonance frequency, the model slightly underpredicts 
the measurements. It is worth noticing that the Forchheimer coefficients are obtained for the MPP resonance frequency. It is suggested that Forchheimer frequency-dependent coefficients give fairly precise results.

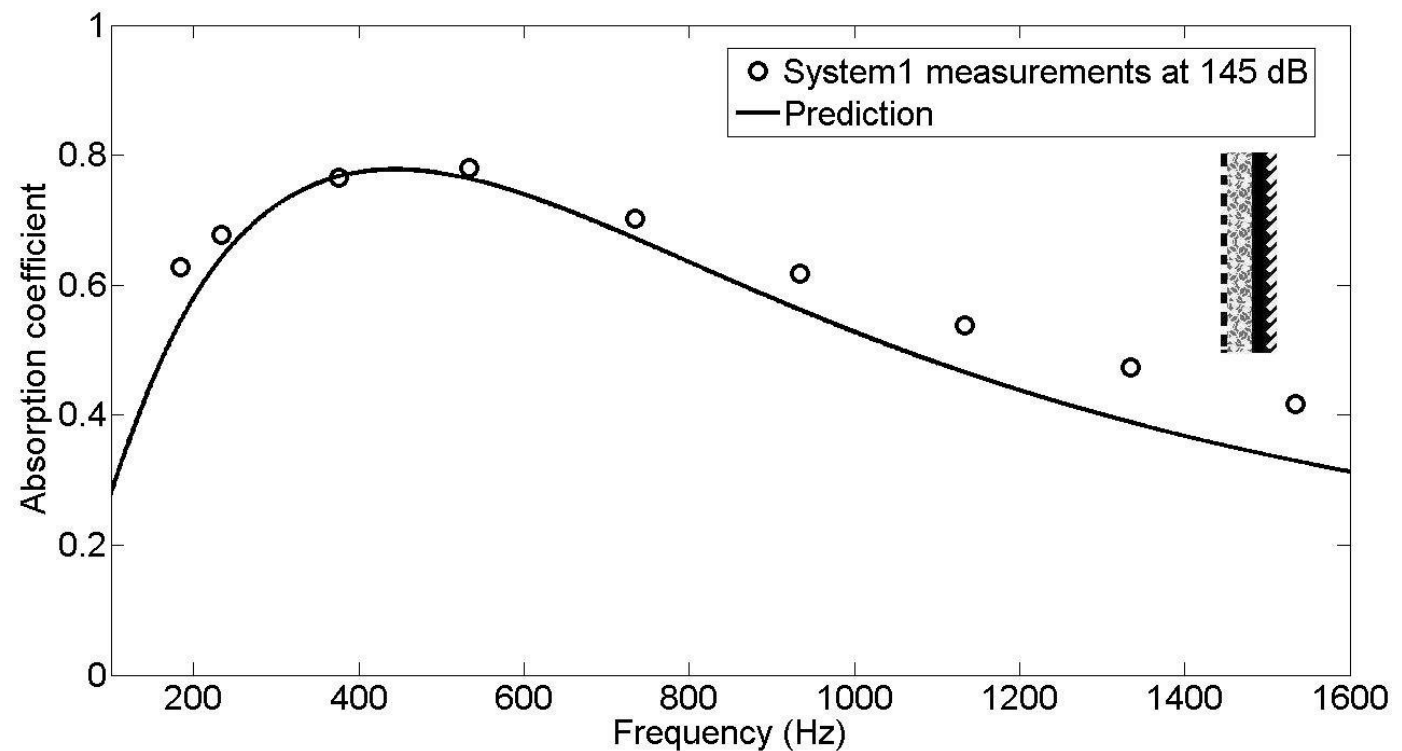

Fig. 6: Absorption coefficient and impedance results as a function of frequency for System1 with no air gap before the rigid wall. Incident pressure level $L_{p}=145 \mathrm{~dB}$ (which corresponds to $R e_{p}=492.5$ ).

Figure 7 presents the measurements and prediction results of the absorption coefficient results as a function of frequency for system\#2 with $57 \mathrm{~mm}$ air cavity before the rigid wall for an incident pressure level $L_{p}$ of $142 \mathrm{~dB}$ (which corresponds to $R e=2.22$ ). It is observed that the measurement and prediction are in good agreement.

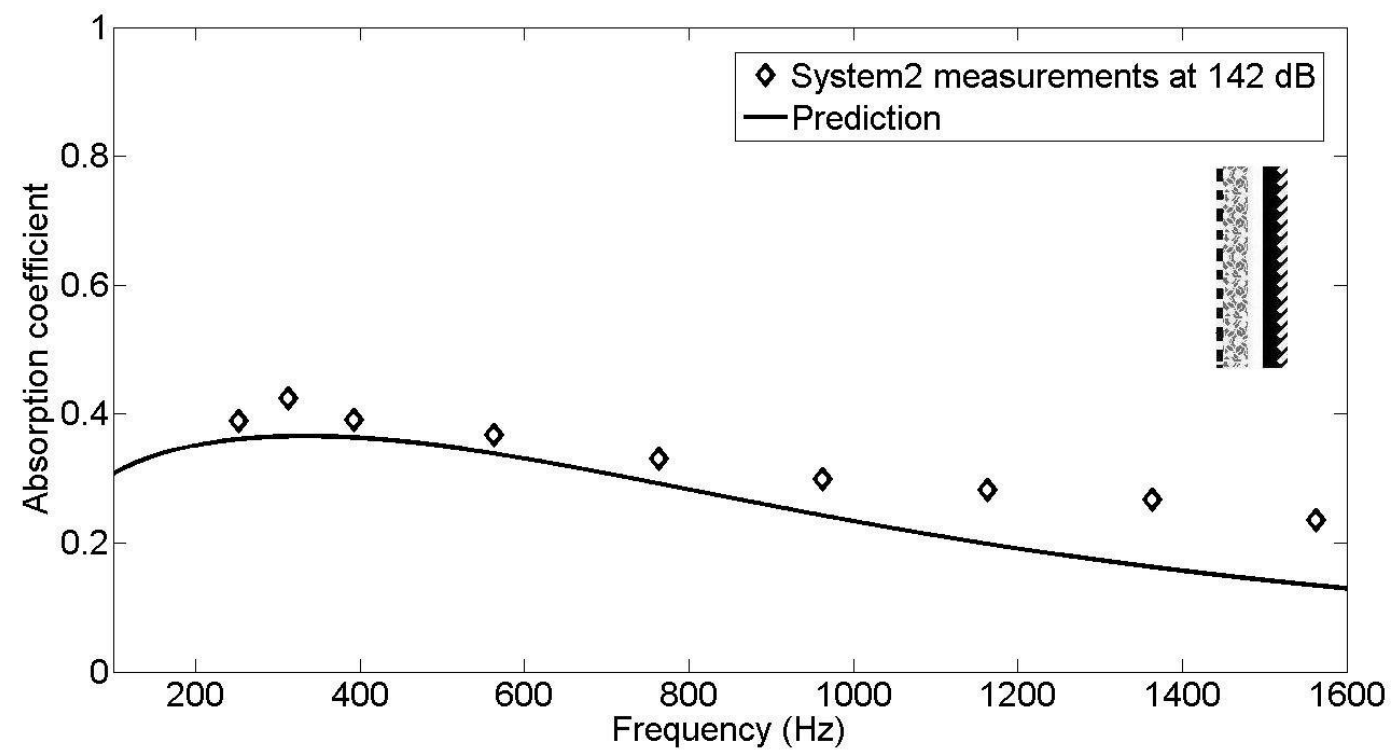

Fig. 7: Absorption coefficient impedance results as a function of frequency for System\#2 with 57 mm air cavity before the rigid wall. Incident pressure level $L_{p}=142 \mathrm{~dB}$ (which corresponds to $R e=2.22$ ).

\section{Conclusion}

In this study, an equivalent fluid approach is used to predict the absorption coefficient of Micro-Perforated Plates (MPP) backed by a porous material under high sound excitations. The coupling between both materials is performed using the Transfer Matrix Method (TMM). To account for the nonlinear regime effects, the resistivity of each layer is modified following the Forchheimer law. Two MPP samples of different thicknesses, hole diameters and open area ratios and two classical porous materials (polymeric foam and fibrous material) were built and tested in three particular cases: the case of the MPP alone backed by an air cavity and a rigid wall, the case of the MPP backed by the porous 
material and the rigid wall without any airspace and finally the case of the MPP backed by the porous material and the rigid wall with an airspace between the porous material and the rigid wall. The comparison between the acoustic measurements and the equivalent fluid model shows that for these assemblies, a modification of the resistivity following the Forchheimer law can be used to predict the absorption coefficient under high sound excitations (also known as the nonlinear regime). Fairly good results are obtained by the model. It was also demonstrated that the porous material behind the micro-perforated plate causes an increase of the system resistance which results into a shift of the limit Reynolds number (Reynolds number value after which the maximum of absorption decreases with the increase of sound level) towards the low Reynolds number ranges.

For the different configurations studied, the model and the measurements are in very good agreement. The fluid equivalent approach was used to model this assembly (MPP backed by a porous material). The next step of the work would be to carry out the same investigation using the Biot elastic approach under high sound excitations which may seem appropriate to model the case where the porous material vibrations is not negligible.

\section{Acknowledgements}

The support grant for this work was provided by the Conseil Régional de Bourgogne during the first author stay in Nevers.

\section{References}

[1] AL-Rahman L.A., Raja I. R., Rahman R. A., "Attenuation of noise by using absorption materials and barriers: A review", International Journal of Engineering and Technology, Vol. 2 (7) (2012), pp.1207-1217.

[2] Davern, W. A., "Perforated facings backed with porous materials as sound absorbers", Applied Acoustics, Vol.10, (1977), pp. 85-112.

[3] Congyun, Z., Qibai, H., "A method for calculating the absorption coefficient of multilayer absorbent using the electro-acoustic analogy", Applied Acoustics, Vol.66, (2005), pp.879-887.

[4] Lee, I. L., Selamet, A., Huff, N. T., "Acoustic impedance of perforations in contact with fibrous material", Journal of Acoustical Society of America, Vol.119, (2006), pp.2785-2797.

[5] Maa, D. Y., "Potential of Micro-perforated panel absorber", Journal of Acoustical Society of America, Vol.104, (1998), pp.2861-2866.

[6] Lee, F.-C., Chen, W.-H., "Acoustic transmission analysis of multi-layer absorbers", Journal of Sound and Vibration, Vol.248, (2001), pp.621634.

[7] Bolton, J. S., Shiau, N.-M., Kang, Y. J., "Sound transmission through multi-panel structures lined with elastic porous materials", Journal of Sound and Vibration, Vol.191, (1996), pp.317-347.

[8] Ver, I. L., Beranek, L. L., Noise and vibration control engineering: Principles and applications, John Wiley Sons Inc., Chap. 8, (2006), pp:215277.

[9] Ingard, U., "On the Theory and Design of Acoustic Resonators”, Journal of Acoustical Society of America, Vol.25, No.6, (1953), pp.10371061 .

[10] Allard, J. F., Atalla N., Propagation of Sound in Porous Media: modeling sound absorbing materials, Elsevier $2^{\text {nd }}$ Ed., (2009), chap 9.

[11] Atalla, N., Sgard, F., "Modeling of perforated plates and screens using rigid frame porous models", Journal of Sound and Vibration, Vol.303, (2007), pp.195-208.

[12] Howe, M. S., "On the theory of unsteady high Reynolds number flow through a circular aperture", Proceedings of the Royal Society of London, Series A 366, (1979), pp.205-223.

[13] Tam, C. K. W., Kurbatskii, A., Ahuja, K. K., Gaeta, R. J. Jr., "A numerical and experimental investigation of the dissipation mechanisms of resonant acoustic liners", Journal of Sound and Vibration, Vol.245, (2001), pp.545-557.

[14] Eldredge, J. D., Bodony, D. J., Shoeybi, M., "Numerical investigation of the acoustic behavior of a multi-perforated liner", Am. Inst. Aeron. Astron. No.2007-3683, - (2007).

[15] Ingard, U., Ising, H., "Acoustic nonlinearity of an orifice”, Journal of Acoustical Society of America, Vol.42, (1967), pp.6-17.

[16] Hersh, A. S., Rogers, T., "Fluid mechanical model of the acoustic impedance of small orifices", U.S. NASA Report No. CR-2682 (1976).

[17] Melling, T. H., "The acoustic Impedance of perforates at medium and high sound pressure levels", Journal of Sound and Vibration, Vol.29, No.1, (1973), pp.9-12.

[18] Cummings, A., "Acoustic nonlinearity and power losses at orifices", Am. Inst. Aeron. Astron. 22, 786-792 (1984).

[19] Tayong, R., Dupont, T., Leclaire, P., "On the variations of acoustic absorption peak with particle velocity in micro-perforated panels at high level of excitation", Journal of Acoustical Society of America, Vol.127, No.5, (2010), pp.2875-2883.

[20] Biot, M. A., "Theory of propagation of elastic waves in a fluid saturated porous solid I: low-frequency range", Journal of Acoustical Society of America, Vol.28, (1956), pp.168-178.

[21] Biot, M. A. "Theory of propagation of elastic waves in a fluid saturated porous solid II: higher frequency range", Journal of Acoustical Society of America, Vol.28, (1956), pp.179-191.

[22] Lauriks, W., Mees, P., Allard, J. F., "The Acoustic transmission through layered systems", Journal of Sound and Vibration, Vol.155, (1992), pp.125-132.

[23] Forchheimer, P., "Wasserbewegung durch boden", Zeit. Ver. Deutsch. Ing. 45, 1781-1788, (1901).

[24] Hassanizadeh, S. M, Gray, W. G., "High velocity flow in porous media", Transp. Porous Media 2, 521-531 (1987).

[25] Auregan, Y., Pachebat, M., "Measurement of the nonlinear behavior of acoustical rigid porous materials", Physics of Fluids, Vol.11, No.6, (1999), pp.1342-1345.

[26] Ingard, U., Labate, S., "Acoustic circulation effects and the nonlinear impedance of orifices", Journal of Acoustical Society of America, Vol.22, No.2, (1940), pp.211-218. 
[27] Barak, A. Z., "Comments on high velocity flow in porous media by Hassanizadeh and Gray", Transp. Porous Media 2, 533 (1987).

[28] Maa, D. Y., "Micro-perforated panel at high sound intensity", Proceedings of InterNoise, Yokohama - Japan (1944).

[29] Umnova, O., Attenborough, K, Standley, E., Cummings, A., "Behavior of rigid porous layers at high levels of continuous acoustic excitation: Theory and experiment", Journal of Acoustical Society of America, Vol.114, (2003), pp.1346-1356.

[30] McIntosh, J. D., Lambert, R. F., "Nonlinear wave propagation through rigid porous materials. I:Nonlinear parametrization and numerical solutions", Journal of Acoustical Society of America, Vol.88, No.4, (1990), pp.1939-1949.

[31] Chung, J. D., Blaser, D. A., "Transfer function method of measuring in-duct acoustic properties. I Theory”, Journal of Acoustical Society of America, Vol.68, No.3, (1980), pp.907-913.

[32] Maa, D.-Y., Liu, K., "Nonlinear standing waves: Theory and experiments", Journal of Acoustical Society of America, Vol.98, No.5, (1995), pp.2753-2763

[33] Dalmont, J-.P., ”Acoustic impedance measurement. part I: a review", Journal of Sound and Vibration, Vol.243, No.3, (2001), pp.441-459. 